\title{
Emergency Strategies of Closed-Loop Supply Chain with Single Retailer Recycling under Demand Disruptions
}

\author{
Jian Cao $\mathbb{D},{ }^{1,2}$ Yuting Yan $\left(\mathbb{D},{ }^{1}\right.$ Lingyuan Wang $\left(\mathbb{D},{ }^{3}\right.$ Xihui Chen $\mathbb{D}^{1},{ }^{1}$ Xuemei Zhang $\mathbb{D}^{1},{ }^{1}$ \\ and Gengui Zhou $\mathbb{D}^{1}$
}

\author{
${ }^{1}$ School of Management, Zhejiang University of Technology, Hangzhou 310023, China \\ ${ }^{2}$ Center for Global \& Regional Environmental Research, The University of Iowa, Iowa City 52242, USA \\ ${ }^{3}$ School of Business, Zhejiang Industry Polytechnic College, Shaoxing 312000, China
}

Correspondence should be addressed to Lingyuan Wang; lingyuan_wang@foxmail.com

Received 6 December 2019; Accepted 20 January 2020; Published 22 February 2020

Academic Editor: Maria Alessandra Ragusa

Copyright (c) 2020 Jian Cao et al. This is an open access article distributed under the Creative Commons Attribution License, which permits unrestricted use, distribution, and reproduction in any medium, provided the original work is properly cited.

The uncertainty caused by emergencies will influence the normal operation of the supply chain. Considering demand disruptions, a closed-loop supply chain consisting of one manufacturer and two competing retailers based on decentralized decision-making is considered. In the supply chain, one retailer recovers end-of-life products while the other does not. Analytic results show that, when the disturbance of demand occurs, the manufacturer and retailers adjust the wholesale price and retail prices of products according to the direction of the market demand disruptions. Under demand disruptions, the retailer who participates in recovering can gain more profits, especially in the case of the positive disruption. Theoretic and pragmatic references for the emergency decision-making of closed-loop supply chain enterprises are provided.

\section{Introduction}

Enterprises are paying more attention than ever to the Closed-Loop Supply Chain (CLSC) in response to governmental regulation and public concerns on environmental pollution and resource consumption. CLSC management has been a popular item on agendas of various industries in the United States since 2005, including automobile, refrigerator compressor, electronic instruments, machinery manufacturing, office appliances, tires, cartridges, industrial valves, and so on. In the past decade, the task of developing CLSC in China has been accepted as a vital need for enterprises. The Chinese government has implemented the Circular Economy Promotion Law which underlines the CLSC management since 2009, and revised it in 2018.

Actually, enterprises participating in CLSC operation can not only win the market share and considerable profits but also have a good impact on the whole ecological environment [1-4]. For instance, Xerox Corporation provides prepaid mailboxes so that customers can easily return their used copy or print cartridges to Xerox without any costs [5].
According to the analysis report of the American Iron and Steel Association, remanufacturing of scrap steel products in the United States has achieved remarkable results: saving energy by $47 \%-74 \%$, reducing air pollution by $86 \%$, reducing water pollution by $76 \%$, reducing solid waste by $97 \%$, and saving water by $40 \%$ [6].

Academic research on CLSC has been quite rich, but most of the literature still focused on the operation of CLSC under stable demand. With the increasingly rapid economic globalization, once an unexpected event interferes with a node in the supply chain, it can easily affect the operation of other parties in the supply chain. For instance, in 2008, the global economic crisis led to a sharp reduction in orders in the industrial chain and a decline in demand from downstream producers, which directly led to a cliff-like decline in supplier output. In 2011, the earthquake of 9.1 magnitude that took place 321 miles northeast of Tokyo at a depth of 15.2 miles, which is the largest earthquake even to hit Japan, caused a shortage of auto parts exports, which led to a substantial increase in the manufacturing costs of several major Japanese joint venture car enterprises located in China. 
Supply chain emergencies refer to risks caused by exogenous events and disruption of normal activities of enterprises on the chain [7]. By studying the irregularity and seriousness of emergencies from two aspects, including possibility and impact of supply chain risk management, it can be concluded that emergencies have the characteristics of low probability of occurrence and high impact consequences $[8,9]$. In this situation, enterprises are required to quickly formulate emergency decision-making plans when supply chains are disrupted.

This paper explores a closed-loop supply chain with one manufacturer and two retailers. One of the retailers participates in waste product recovering. The manufacturer decides the wholesale price as well as the compensation price, while the retailer chooses the retail price, recovering rate, and order quantity. The objective of this paper is to investigate the impact of demand disturbance on supply chain members and to analyze how manufacturers and retailers with dynamic game relationships make optimal emergency decisions when some emergent events occur. A game theoretic model of a closed-loop supply chain with one manufacturer and two retailers is established to solve the following problems.

(i) What are the optimal decisions of supply chain members when demand disruption occurs?

(ii) Is there a direct relationship between retailers' participation in recycling and their market competitiveness under demand disruption?

(iii) What are the influences of different market demand disruptions on the decision-making of CLSC members?

The contributions of this paper are summarized as follows. Firstly, the impact of demand disruption on optimal CLSC decisions and profits is examined, when the dynamic game between retailers is considered simultaneously. Secondly, the optimal decisions are analyzed from the perspectives of both the manufacturer and the two retailers. Thirdly, practical suggestions on how to maintain the market competitiveness of retailers under demand disruption are put forward. The remainder of this paper is organized as follows: Section 2 reviews the related literature in the field of supply chain management. Section 3 establishes the model and obtains the optimal results under stable condition. Section 4 discusses CLSC optimal decisions under demand disruption. Section 5 provides numerical examples to examine the propositions and shed practical implications. Finally, the conclusions and future research directions are summarized in Section 6.

\section{Literature Review}

In recent years, scholars have carried out research on different types of CLSC problems. Savaskan et al. [5] investigate how reverse channel choice affects the forward channel decisions and the used-product return rates by considering three different modes in the collection channels of manufacturers, retailers, and third party participation in recycling.
Yang et al. [10] developed a model of a general CLSC network, which formulates and optimizes the equilibrium state of the network using variational inequalities. Shi et al. [11] studied the production planning problem for a multiproduct closed-loop system, in which the manufacturer has two channels for supplying products: producing brand-new products and remanufacturing returns into new ones. They maximize the manufacturer's expected profit by jointly determining the production quantities of brand-new products, the quantities of remanufactured products, and the acquisition prices of the used products, subject to a capacity constraint. Choi et al. [12] investigated a CLSC which consists of a retailer, a collector, and a manufacturer, and examined the performance of different CLSCs under different channel leaderships. Through a systematic comparison, they find that the retailer-led model gives the most effective CLSC. Huan et al. [13] considered optimal strategies of a CLSC with a dual recycling channel, within which the manufacturer sells products via the retailer in the forward supply chain, while the retailer and the third party competitively collect used products in the reverse supply chain. Abbey et al. [14] studied empirically to investigate consumer perceptions of remanufactured consumer products in the CLSC. The results indicate that discounting had a consistently positive, linear effect on remanufactured product attractiveness. Moreover, consumers who consider remanufactured products green typically found remanufactured products significantly more attractive. De Giovanni et al. [15] considered a dynamic CLSC with one manufacturer and one retailer, both of which are investing in a product recovery program to increase the return rate of previously purchased products. Gaur et al. [16] developed an integrated optimization model for Closed-Loop Supply Chain Configuration (CLSCC) and presented a real-world case study of a battery manufacturer. They discussed the key observations and managerial implications drawn from the computational experiments. The significant outcomes of the study suggest that the performance of the firm's base case integrated CLSCC model is significantly better than the current supply chain model. Reimann et al. [17] focused on the link between remanufacturing and the opportunity to lower the variable remanufacturing cost via process innovation. Their analysis also shows that compared with integrated supply chain, decentralized supply chain is more likely to take up manufacturing, especially when the cost of process innovation is sufficiently high.

From above, most literature assume that the internal and external environments of CLSC remain stable. In actual operations, however, any kinds of unexpected events (market or human factors, such as natural disasters, terrorist attacks, national conflicts, and economic turbulence) will make the optimal decision-making under stable conditions unfeasible. Therefore, the research on dealing with supply chain emergencies has attracted wide attention of scholars, and disturbance management emerged. Disruption management was first proposed by Clausen et al. [18] and was initially applied in the fields of aviation as well as supply chain management. Qi et al. [19] considered the impact of emergency management on supply chain based on the work 
of Clausen and opened up a new avenue for supply chain management. Kleindorfer and Saad [20] investigated risks arising from natural disasters, strikes, and economic chaos, as well as acts of purposeful agents, including terrorists. The implications for the design of management systems intended to cope with supply chain disruption risks discussed. Xiao et al. [21] studied the impact of two price-sensitive, partially replaceable retailers who bear the production deviation cost caused by accidents on production planning. Hou et al. [22] explore the contract coordination mechanism between the manufacturer and the two suppliers in the case of demand uncertainty and supply risk. Huang et al. [23] provided a two-period pricing and production decision model in a onemanufacturer-one-retailer dual-channel supply chain and examined how to adjust the prices and the production plan to meet the maximal profit under a disruption scenario. Huang et al. [24] showed how the members of a centralized and decentralized dual-channel supply chain adjust their production plans according to the disturbance of production costs. Giri and Sharma [25] considered a CLSC inventory system involving a single-manufacturer and a single-retailer with stochastic market demand and random return of used item. They proposed the optimal quantity of finished products manufactured from raw materials to maximize the total profit of the integrated system. Huang and Wang [26] investigated cost disruptions of new and remanufactured products in a CLSC where a manufacturer and a third-party collector recycle used products through online-recycling and offline-recycling channels, respectively. Huang and Wang [27] studied disruptions of market demand and supply quantity of used products in a CLSC where the manufacturer licenses the third party to undertake remanufacturing activities.

To the best of our knowledge, very few research results on emergency strategies of CLSC in a single retailer recycling under demand disruption have been achieved. We attempt to fill the research gap by including such concerns, which highlights the originality and contributions of this paper. We construct a CLSC model based on decentralized decisionmaking, which is composed of one manufacturer and two competing retailers. One retailer participates in the recovering of waste products, while the other does not. This paper discusses the emergency decision-making of supply chain members under demand disruptions. The research work could provide some references for the emergency decisionmaking of CLSC enterprises.

\section{The Basic Model}

3.1. Model and Symbolic Description. The supply chain consisting of a single manufacturer and two competing retailers is considered in this paper. The manufacturer dominates in the production of two products: new products and remanufactured products. The remanufactured products, having the same quality as the new ones, are processed from the waste products recovered by the retailer. Two competing retailers are in a follower position in the supply chain operation. They get new and remanufactured products at the same wholesale price from the manufacturer. Each retailer independently decides the price for two kinds of products; hence, different prices are set according to two retailers. We suppose that waste products are recovered by only one of the retailers (retailer 1). The basic model is shown in Figure 1 with all notations introduced in Table 1.

3.2. Closed-Loop Supply Chain Decisions under Stable Condition. The profit functions of the manufacturer and two retailers are obtained as follows:

$$
\begin{aligned}
\pi_{M}(w, b)= & {\left[w-c_{n}+\tau(\Delta-b)\right]\left[2 \phi+(\beta-1)\left(p_{1}+p_{2}\right)\right], } \\
\pi_{R_{1}}\left(p_{1}, \tau\right)= & \left(p_{1}-w\right)\left(\phi-p_{1}+\beta p_{2}\right)+[2 \phi+(\beta-1) \\
& \left.\cdot\left(p_{1}+p_{2}\right)\right] b \tau-h \tau^{2} \\
\pi_{R_{2}}\left(p_{2}\right)= & \left(p_{2}-w\right)\left(\phi-p_{2}+\beta p_{1}\right) .
\end{aligned}
$$

In the decentralized decisions of the closed-loop supply chain where the manufacturer acts as the Stackelberg leader, the solution of the following proposition (see Appendix A for proof) is obtained by using backward induction method (the superscript ${ }^{*}$ represents the optimal solution). For convenience, we define

$$
\begin{aligned}
& A=c_{n}(\beta-1), \\
& B=2 h\left(2+\beta-\beta^{2}\right)-(1-\beta) \Delta^{2}, \\
& C=h(1+\beta)^{2}, \\
& D=(\beta-1) \Delta^{2} .
\end{aligned}
$$

Proposition 1. In the closed-loop supply chain where a single retailer is responsible for recovering the used products, the optimal strategies of the manufacturer and retailers are given as follows:

(a) The wholesale price $\left(w^{*}\right)$, compensation price $\left(b^{*}\right)$, and profit $\left(\pi_{M}^{*}\right)$ are obtained as

$$
\begin{aligned}
w^{*} & =\frac{2 C(2-\beta)(\phi-A)+D\left[c_{n}(\beta-1)^{2}+\phi+3 \beta \phi\right]}{2\left(1-\beta^{2}\right) B}, \\
b^{*} & =\frac{\Delta}{\beta+1}, \\
\pi_{M}^{*} & =\frac{h(1+\beta)(A+\phi)^{2}}{(1-\beta) B} .
\end{aligned}
$$

(b) The retail price $\left(p_{1}^{*}\right)$, recovering rate of waste products $\left(\tau^{*}\right)$, and profit $\left(\pi_{R_{1}}^{*}\right)$ of retailer 1 are shown as 


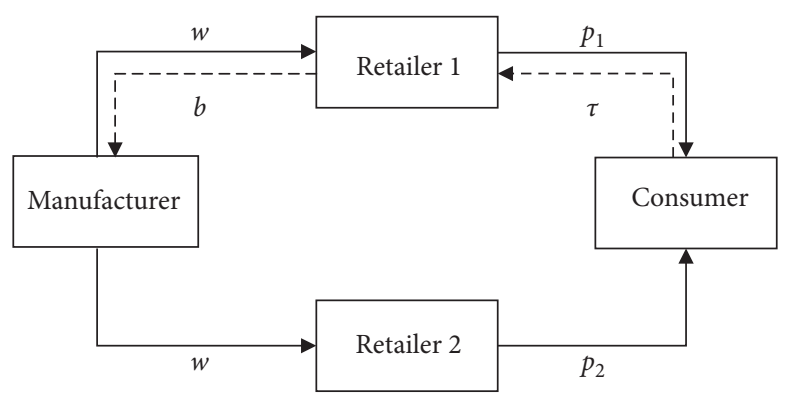

Forward logistics

---- Reverse logistics

FIgURE 1: CLSC models with single retailer in recycling.

TAble 1: Notations.

\begin{tabular}{|c|c|}
\hline Symbol & Description \\
\hline \multicolumn{2}{|c|}{ Parameters } \\
\hline $\mathrm{cn}$ & Manufacturing cost of unit new product \\
\hline $\mathrm{cr}$ & Manufacturing cost of unit remanufactured product \\
\hline$\Delta$ & Unit cost savings from remanufacturing, $\Delta=\mathrm{cn}-\mathrm{cr} \geq 0$ \\
\hline$\phi$ & Market size \\
\hline$\varphi$ & Disruption of market demand, $\phi+\varphi \geq 0$ \\
\hline$\beta$ & Substitution rate of competing retailers selling the same product, $\beta \in[0,1]$ \\
\hline \multicolumn{2}{|c|}{ Variables and functions } \\
\hline$w$ & Wholesale price of unit product sold by manufacturer to retailers \\
\hline$\tau$ & Recycling rate of waste products, $\tau \in[0,1]$ \\
\hline pi & Retail price of unit product sold by retailers to consumers, $i=1,2$ (represent retailer 1 and retailer 2 , respectively) \\
\hline$b$ & Unit compensation price provided by the manufacturer to retailer 1 for recycling waste products, $b \in[0, \Delta]$ \\
\hline$I$ & Fixed investment cost of recycling waste products by retailer $1, I=h \tau 2, h$ is a scale parameters \\
\hline$q_{i}$ & Market demand of the retailer $i, q_{i}=\phi-p_{i}+\beta p_{j}, i, j=1,2$ \\
\hline$Q$ & Market demand of both products, $Q=q_{1}+q_{2}$ \\
\hline$\pi k$ & Profit function, $k=M, \mathrm{R} 1, \mathrm{R} 2$ (represent manufacturer, retailer 1 and retailer 2, respectively) \\
\hline
\end{tabular}

$$
\begin{aligned}
& p_{1}^{*}=\frac{2 C(2+\beta)[(3-2 \beta) \phi-A]+D\left[c_{n}(\beta-1)^{2}-\left(5+5 \beta+2 \beta^{2}\right) \phi\right]}{2 B\left(1-\beta^{2}\right)(2+\beta)}, \\
& \tau^{*}=\frac{\Delta[A+\phi]}{B}, \\
& \pi_{R_{1}}^{*}=\frac{[A+\phi]^{2}\left\{4 C(2+\beta)\left[h\left(\beta^{2}+3 \beta+2\right)+3 \Delta^{2}\right]-\Delta^{2}\left[4 h(1+\beta)(2+\beta)^{2}+(\beta-1)^{2}(3+\beta) \Delta^{2}\right]\right\}}{4\left(2+\beta^{2}\right)(1+\beta) B^{2}} .
\end{aligned}
$$

(c) The retail price $\left(p_{2}^{*}\right)$ and profit $\left(\pi_{R_{2}}^{*}\right)$ of retailer 2 are displayed as

$$
\begin{aligned}
& p_{2}^{*}=\frac{2 C(2+\beta)[(3-2 \beta) \phi-A]+D\left[c_{n}(\beta-1)^{2}+\left(3+7 \beta+2 \beta^{2}\right) \phi\right]}{2 B\left(1-\beta^{2}\right)(2+\beta)}, \\
& \pi_{R_{2}}^{*}=\frac{(A+\phi)^{2}\left[2 h\left(2+3 \beta+\beta^{2}\right)+D\right]^{2}}{4\left(2+\beta^{2}\right) B^{2}} .
\end{aligned}
$$


(d) The total sales of the two retailers are presented as $q^{*}=(2 h(1+\beta)(A+\phi)) / B$.

\section{Closed-Loop Supply Chain Decision under Demand Disruption}

When the disturbance of demand occurs, the market size $\phi$ is turned into $\phi+\varphi$, while the market demand of the retailer is changed to $\bar{q}_{i}=\phi+\varphi-p_{i}+\beta p_{j}(i, j=1,2$, “-” represents the results of market disruption). When $\varphi>0$, the optimal strategy under stable condition cannot meet the demand due to the sudden expansion of the market; therefore, additional production costs $\lambda_{1}\left(\lambda_{1}>0\right)$ will be generated. When $\varphi<0$, the supply chain will suffer additional processing costs $\lambda_{2}\left(\lambda_{2}>0\right)$ due to abrupt decrease of market size. It is assumed that the additional production or processing costs are borne by the manufacturer.

Based on the basic model and abovementioned assumptions, profit functions of the manufacturer and retailers under demand disruptions are obtained as follows.

$$
\begin{aligned}
\bar{\pi}_{M}= & {\left[2(\phi+\varphi)+(\beta-1)\left(\bar{p}_{1}+\bar{p}_{2}\right)\right]\left[\bar{w}-c_{n}+\bar{\tau}(\Delta-\bar{b})\right]-\lambda_{1}\left\{2(\phi+\varphi)+(\beta-1)\left(\bar{p}_{1}+\bar{p}_{2}\right)-\frac{2 h(1+\beta)\left[c_{n}(\beta-1)+\phi\right]}{h\left(4+2 \beta-2 \beta^{2}\right)+(\beta-1) \Delta^{2}}\right\}^{+} } \\
& -\lambda_{2}\left\{\frac{2 h(1+\beta)\left[c_{n}(\beta-1)+\phi\right]}{h\left(4+2 \beta-2 \beta^{2}\right)+(\beta-1) \Delta^{2}}-2(\phi+\varphi)+(\beta-1)\left(\bar{p}_{1}+\bar{p}_{2}\right)\right\}^{+}, \\
\bar{\pi}_{R_{1}}= & \left(\phi+\varphi-\bar{p}_{1}+\beta \bar{p}_{2}\right)\left(\bar{p}_{1}-\bar{w}\right)+\left[2(\phi+\varphi)+(\beta-1)\left(\bar{p}_{1}+\bar{p}_{2}\right)\right] \bar{\tau}-h \bar{\tau}^{2}, \\
\bar{\pi}_{R_{2}}= & \left(\phi+\varphi-\bar{p}_{2}+\beta \bar{p}_{1}\right)\left(\bar{p}_{2}-\bar{w}\right) .
\end{aligned}
$$

Lemma 1. Under demand disruption caused by emergencies, if $\varphi>0$, then $\bar{q}^{*} \geq q^{*}, 1 \geq \bar{\tau}^{*} \geq \tau^{*}$. On the contrary, if $\varphi<0$, then $\bar{q}^{*} \leq q^{*}, \bar{\tau}^{*} \leq \tau^{*} \cdot \bar{q}^{*}$ and $\bar{\tau}^{*}$ are, respectively, the optimal order quantity and recovering rate of waste products.
4.1. Scenario $I(\varphi>0)$. When $\varphi>0$, strategies of retailers in Lemma 1 can be summarized as the following optimization problems.

The proof is given in Appendix B.

$$
\begin{aligned}
& (P 1): \max _{\bar{p}_{1}, \bar{\tau}} \bar{\pi}_{R_{1}}=\left(\phi+\varphi-\bar{p}_{1}+\beta \bar{p}_{2}\right)\left(\bar{p}_{1}-\bar{w}\right)+\left[2(\phi+\varphi)+(\beta-1)\left(\bar{p}_{1}+\bar{p}_{2}\right)\right] \bar{b} \bar{\tau}-h \bar{\tau}^{2} \\
& \text { s.t. } \frac{\Delta\left[c_{n}(\beta-1)+\phi\right]}{h\left(4+2 \beta-2 \beta^{2}\right)+(\beta-1) \Delta^{2}}-\bar{\tau} \leq 0, \quad \bar{\tau}-1 \leq 0, \\
& (P 2): \max _{\bar{p}_{2}} \bar{\pi}_{R_{2}}=\left(\phi+\Delta \phi-\bar{p}_{2}+\beta \bar{p}_{1}\right)\left(\bar{p}_{2}-\bar{w}\right) .
\end{aligned}
$$

The optimal retail prices $\bar{p}_{1}^{*}(\bar{w}, \bar{b})$ and $\bar{p}_{2}^{*}(\bar{w}, \bar{b})$ and recovery rate $\bar{\tau}^{*}(\bar{w}, \bar{b})$ of retailers, which are functions of wholesale price $\bar{w}$ and compensation price $\bar{b}$, can be obtained. The solution process is introduced as follows.
Taking partial derivative of $\bar{\pi}_{R_{1}}$ with respect to $\bar{p}_{1}$ and $\bar{\tau}$ and partial derivative of $\bar{\pi}_{R_{2}}$ with respect to $\bar{p}_{2}$, let $\partial \bar{\pi}_{R_{1}} / \partial \bar{p}_{1}=0, \partial \bar{\pi}_{R_{1}} / \partial \bar{\tau}=0$, and $\partial \bar{\pi}_{R_{1}} / \partial \bar{p}_{2}=0$, we can get 


$$
\begin{aligned}
& \bar{p}_{1}^{*}=-\frac{2 h(2+\beta)(w+\phi+\varphi)+b^{2}(-1+\beta)[w(-1+\beta)+(3+\beta)(\phi+\varphi)]}{(2+\beta)\left[2 h(-2+\beta)+b^{2}(-1+\beta)^{2}\right]}, \\
& \bar{p}_{2}^{*}=\frac{-2 h(2+\beta)(w+\phi+\varphi)+b^{2}\left[w(-1+\beta)^{2}-\left(-1+\beta^{2}\right)(\phi+\varphi)\right]}{(2+\beta)\left[2 h(-2+\beta)+b^{2}(-1+\beta)^{2}\right]}, \\
& \bar{\tau}^{*}=-\frac{2 b[w(-1+\beta)+\phi+\varphi]}{2 h(-2+\beta)+b^{2}(-1+\beta)^{2}} .
\end{aligned}
$$

By substituting equations (9) and (11) into equation (4), the decision-making problem of the manufacturer can be transformed into the following optimization problem.

$$
\begin{aligned}
(P 3): \max _{\bar{w}, \bar{b}} \bar{\pi}_{M}=\left\{2(\phi+\varphi)+(\beta-1)\left[\bar{p}_{1}^{*}(\bar{w}, \bar{b})+\bar{p}_{2}^{*}(\bar{w}, \bar{b})\right]\right\}\left[\bar{w}-c_{n}+\bar{\tau}^{*}(\bar{w}, \bar{b})(\Delta-\bar{b})\right] \\
\quad-\lambda_{1}\left\{2(\phi+\varphi)+(\beta-1)\left(\bar{p}_{1}^{*}(\bar{w}, \bar{b})+\bar{p}_{2}^{*}(\bar{w}, \bar{b})\right)-\frac{2 h(1+\beta)\left[c_{n}(\beta-1)+\phi\right]}{h\left(4+2 \beta-2 \beta^{2}\right)+(\beta-1) \Delta^{2}}\right\}, \\
\text { s.t. } \frac{2 h(1+\beta)\left[c_{n}(\beta-1)+\phi\right]}{h\left(4+2 \beta-2 \beta^{2}\right)+(\beta-1) \Delta^{2}}-\bar{q}^{*}(\bar{w}, \bar{b}) \leq 0, \\
\quad \frac{\Delta\left[c_{n}(\beta-1)+\phi\right]}{h\left(4+2 \beta-2 \beta^{2}\right)+(\beta-1) \Delta^{2}}-\bar{\tau}^{*}(\bar{w}, \bar{b}) \leq 0, \\
\bar{\tau}^{*}(\bar{w}, \bar{b})-1 \leq 0 .
\end{aligned}
$$

Equation (13) shows that when the market's demand suddenly occurs positive disruption, the expected order quantity under the stable condition cannot meet the actual order quantity. Equations (14) and (15) show that the recovery rate of retailer 1 under the demand disruption should not be lower than that under the stable condition. We use the backward induction method to solve (P1), (P2), and (P3) and derive the following proposition.

Proposition 2. When $\varphi>0$, the optimal compensation price of the manufacturer under decentralized decision-making is $\vec{b}^{*}=\Delta /(\beta+1)$, the optimal wholesale price and the optimal profit of the manufacturer are, respectively, $\bar{w}^{*}$ and $\bar{\pi}_{M}^{*}$, as shown in Table 2.

As is indicated in Proposition 2, when $\varphi>0$, under different disruptions, the optimal compensation price of the manufacturer remains constant as $\bar{b}^{*}=\Delta /(\beta+1)$, with strong robustness. With the increase of market demand, the manufacturer will suffer additional production costs. So, the manufacturer would improve the wholesale price to compensate extra costs. With the increase of the wholesale price, the retailer must maintain its profits by raising the retail price, to control the excessive increase of market demand. Additionally, the manufacturer's optimal profit increases as the market demand disruption increases.
Proposition 3. When $\varphi>0$, the retailers make strategies to pursue profit maximization after observing the manufacturer's decision. The results are shown in Tables 3 and 4.

When $\varphi \in\left(0,(1-\beta) \lambda_{1}\right)$, the optimal recovery rate of retailer $1 \bar{\tau}^{*}$ and the optimal total orders $\bar{q}^{*}$ are as same as the optimal solution under stable condition. When $\varphi>0$, with the increase of the wholesale price of the manufacturer, retailer 1 can transfer additional production costs to consumers via increased retail price. It can not only compensate for the loss caused by the manufacturer's increasing wholesale price, but also maintains their own profits. When $\varphi>J$, the recovery rate of retailer 1 reaches a maximum value of 1 (that is, all waste products in the market can be effectively recycled). The profit of retailer 1 increases when the demand disruption occurs.

To sum up, under the demand disruption, the retail price of retailer 2 is higher than that of retailer 1. Being in competition with retailer 2 , retailer 1 can get extra profit via recovering the waste products and will increase its market sales by lowering the retail price. In terms of profit, retailer 1 can also get more profits than retailer 2 .

4.2. Scenario $I I(\varphi<0)$. When the demand disruption $\varphi<0$, the profit function of the manufacturer is as follows: 


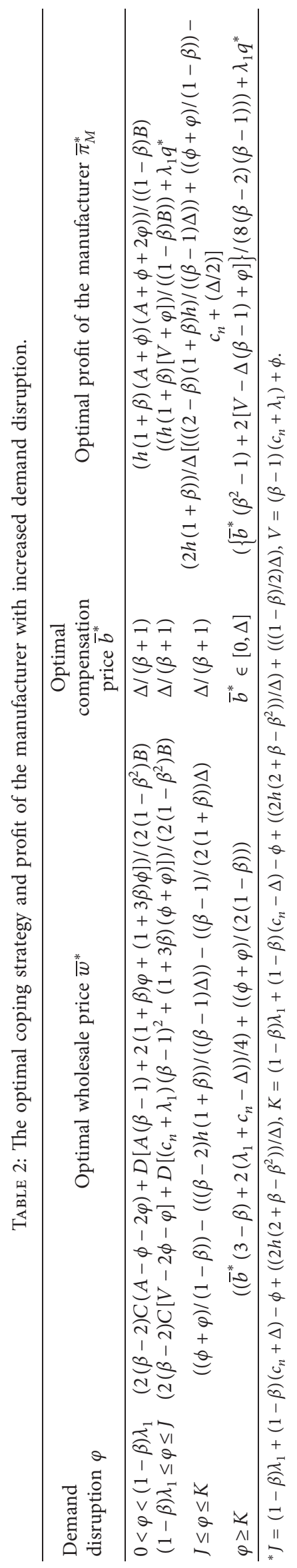




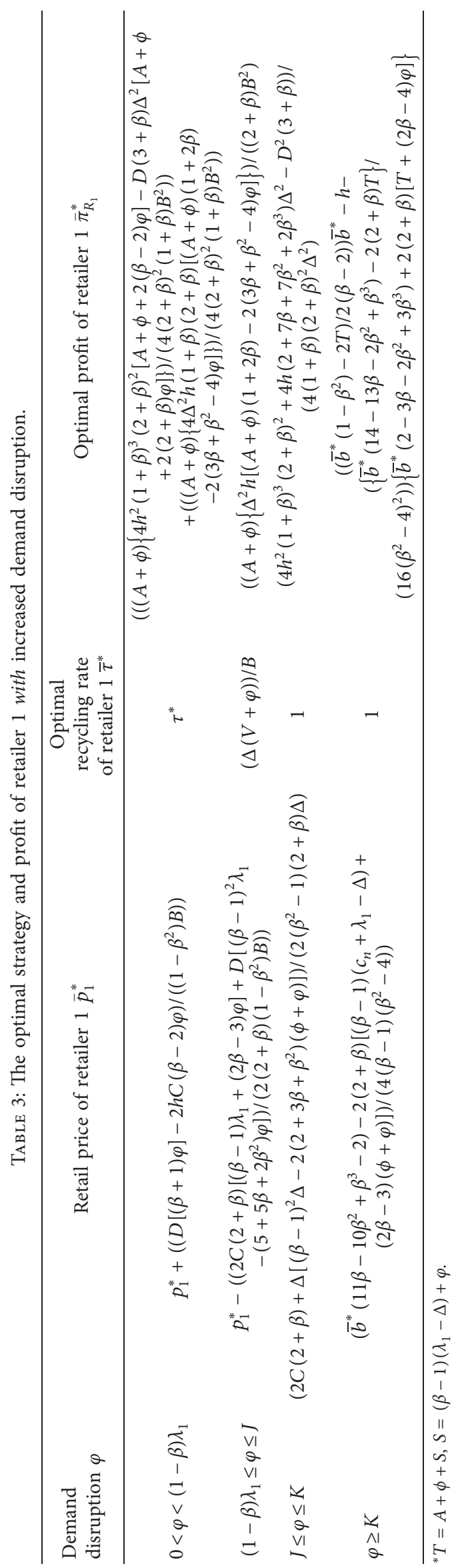




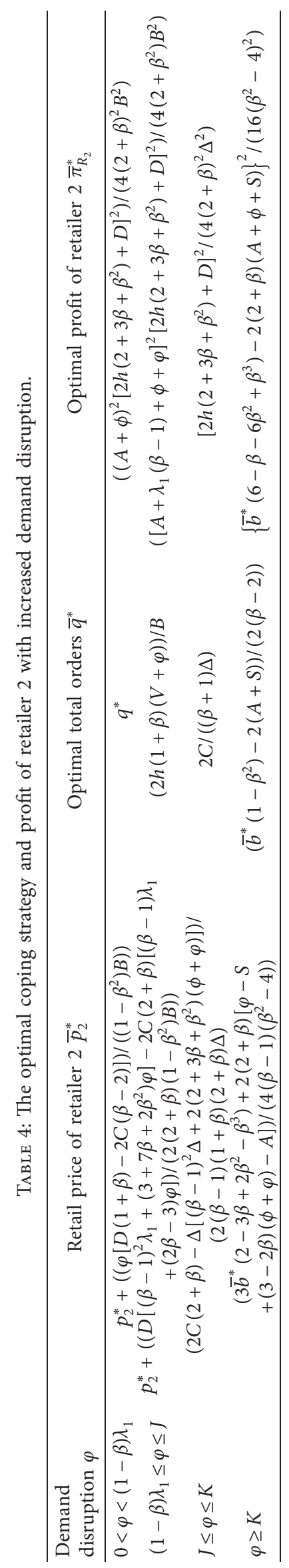




$$
\begin{aligned}
\bar{\pi}_{M}= & {\left[2(\phi+\varphi)+(\beta-1)\left(\bar{p}_{1}+\bar{p}_{2}\right)\right] } \\
& \times\left[\bar{w}-c_{n}+\bar{\tau}(\Delta-\bar{b})\right] \\
& -\lambda_{2}\left\{q^{*}-\left[2(\phi+\varphi)+(\beta-1)\left(\bar{p}_{1}+\bar{p}_{2}\right)\right]\right\} .
\end{aligned}
$$

By using Karush-Kuhn-Tucker (KKT) conditions, the decision-making problem of two retailers can be transformed into the following optimization problems.

$$
\begin{aligned}
& \text { (P4): } \max _{\bar{p}_{1}, \bar{\tau}} \bar{\pi}_{R_{1}}=\left(\phi+\varphi-\bar{p}_{1}+\beta \bar{p}_{2}\right) \times\left(\bar{p}_{2}-\bar{w}\right)+\left[2(\phi+\varphi)+(\beta-1)\left(\bar{p}_{1}+\bar{p}_{2}\right)\right] \bar{b} \bar{\tau}-h \bar{\tau}^{2} \\
& \text { s.t. } \quad \bar{\tau}^{*}(\bar{w}, \bar{b})-\frac{\Delta\left[c_{n}(\beta-1)+\phi\right]}{h\left(4+2 \beta-2 \beta^{2}\right)+(\beta-1) \Delta^{2}} \leq 0, \bar{\tau}(\bar{w}, \bar{b})-1 \leq 0, \\
& \text { (P5): } \max _{\bar{p}_{2}} \bar{\pi}_{R_{2}}=\left(\phi+\varphi-\bar{p}_{2}+\beta \bar{p}_{1}\right) \times\left(\bar{p}_{2}-\bar{w}\right) .
\end{aligned}
$$

Similarly, the decision-making problems of the manufacturer can be transformed into the following optimization problems.

$$
\begin{aligned}
& \text { (P6): } \max _{\bar{w}, \bar{b}} \quad \bar{\pi}_{M}=\left[2(\phi+\varphi)+(\beta-1)\left(\bar{p}_{1}^{*}+\bar{p}_{2}^{*}\right)\right] \times[\bar{w} \\
& \left.-c_{n}+\bar{\tau}^{*}(\Delta-\bar{b})\right]-\lambda_{2}\left\{q^{*}-[2(\phi+\varphi)\right. \\
& \left.\left.+(\beta-1)\left(\bar{p}_{1}^{*}+\bar{p}_{2}^{*}\right)\right]\right\} \\
& \text { s.t. } \quad \bar{q}^{*}(\bar{w}, \bar{b})-\frac{2 h(1+\beta)\left[c_{n}(\beta-1)+\phi\right]}{h\left(4+2 \beta-2 \beta^{2}\right)+(\beta-1) \Delta^{2}} \leq 0, \\
& \bar{\tau}^{*}(\bar{w}, \bar{b})-\frac{\Delta\left[c_{n}(\beta-1)+\phi\right]}{h\left(4+2 \beta-2 \beta^{2}\right)+(\beta-1) \Delta^{2}} \leq 0, \\
& \bar{\tau}(\bar{w}, \bar{b})-1 \leq 0 .
\end{aligned}
$$

Equation (19) indicates that when the market's demand size suddenly shows negative disruption, the expected order quantity under the stable condition exceeds the actual order quantity under demand disruption. Equations (20) and (21) are used to ensure that the constraints of retailer 1 can be effectively satisfied. We use the backward induction method to solve (P4), (P5), and (P6) and derive the propositions as follows.

Proposition 4. When $\varphi<0$, the optimal compensation price of the manufacturer under decentralized decision-making is $\vec{b}^{*}=\Delta /(1+\beta)$, while the optimal wholesale price and the optimal profit of the manufacturer are, respectively, $\bar{w}^{*}$ and $\bar{\pi}_{M}^{*}$, as shown in Table 5.

It can be seen from Proposition 4 that, when $\varphi<0$, under different disruptions, the optimal compensation price of the manufacturer still remains $\bar{b}^{*}=\Delta /(\beta+1)$, with strong robustness. The wholesale price of the manufacturer is inversely proportional to the absolute value of market demand disruption. That is, the larger the decrease of demand is, the lower the wholesale price is. With the reduction of the wholesale price of the manufacturer, the retailer will decrease the retail price, in order to control the excessive reduction of market demand. By substituting the optimal solution of the manufacturer $\bar{b}^{*}$ and $\bar{w}^{*}$ into the profit function of retailers, the optimal solution under the demand disruption can be obtained.

Proposition 5. When $\varphi<0$, with the goal of maximizing their own profits, the retailers develop their own optimal strategy based on the manufacturer's decision. The results are shown in Tables 6 and 7.

When $-(1-\beta) \lambda_{2} \leq \varphi \leq 0$, the optimal recovery rate of retailer $1 \bar{\tau}^{*}$ and the optimal total orders $\bar{q}^{*}$ are the same as the optimal solution under stable condition, with strong robustness. The change of the retail price of retailer $1 \bar{p}_{1}^{*}$ and the wholesale price of the manufacturer $\bar{w}^{*}$ shows consistent trends. Retailers must reduce prices to avoid unsalable products due to the reduction of demand. Therefore, profits of retailers are greatly reduced.

Comparing with Tables 6 and 7, we can see that the retail price of retailer 2 is higher than that of retailer 1 . With the extra profit from the recovery of the waste products, retailer 1's profit is higher than retailer 2's even when the market demand reduces. Therefore, retailers who do not participate in recovering will have less ability to stabilize the market than retailers who participate in recovering waste products under demand disruption.

\section{Numerical Examples}

We further present the numerical examples to examine the theoretical results mentioned above. The basic parameters are set as follows: $\phi=30, c_{n}=5, c_{r}=3, h=15, \beta=0.85$, $\lambda_{1}=2.5, \lambda_{2}=2.5$, and $\varphi \in[-20,24]$. The optimal pricing of two retailers under decentralized decision-making is shown in Figure 2. 


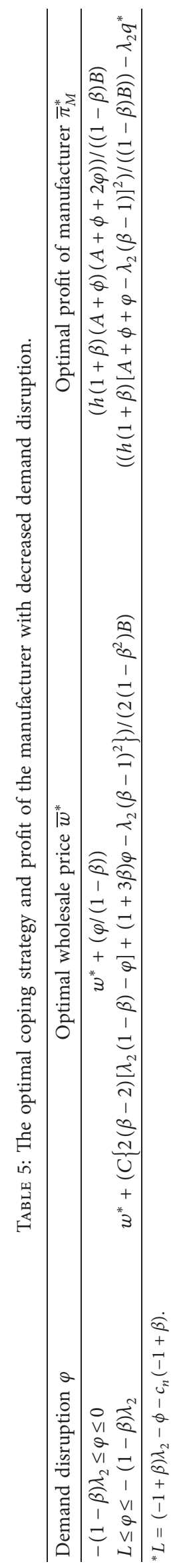




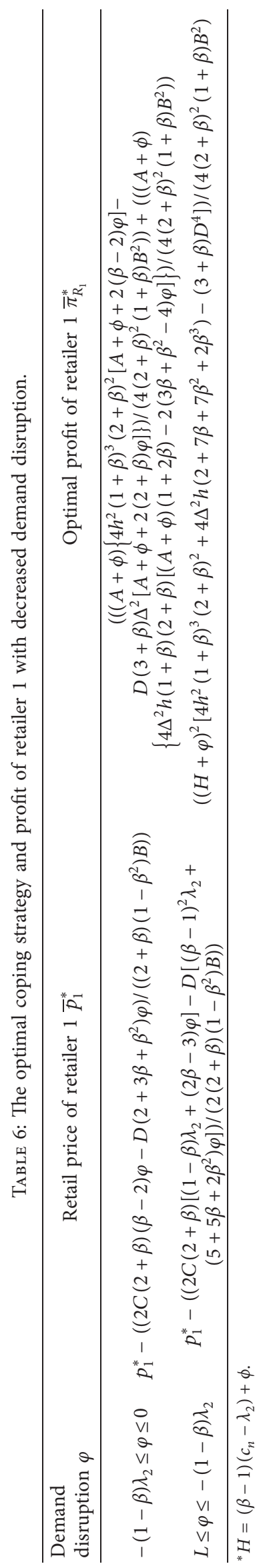




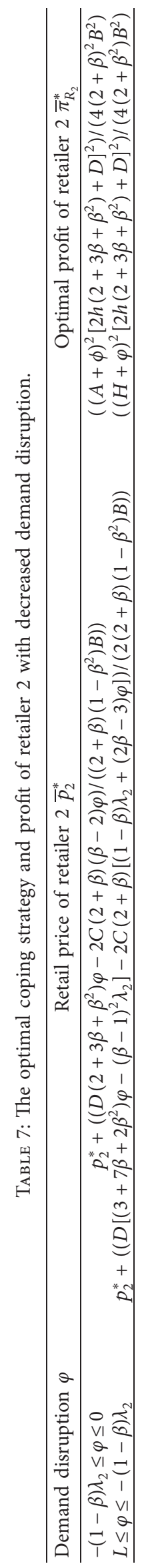




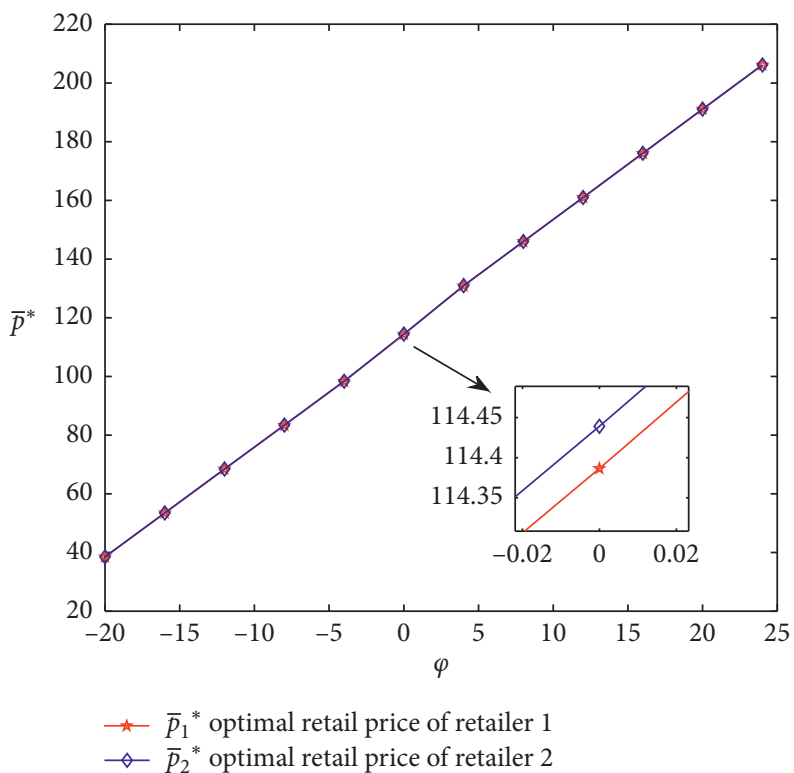

FIgURE 2: Comparison of optimal retail prices between two retailers.

As is shown in Figure 2, both retailers adjust their prices with the variance of demand disruption. The retail price is positively correlated with demand disruption. The price of retailer 1 is always lower than that of retailer 2 regardless of the change of demand disruption. Thus, if only one of the competing retailers participates in recovering waste products, the retailer which adopts this strategy will be more dominant under demand disruption. To consumers, the price of the retailer involved in recovering the waste is more attractive.

Figure 3 shows the profits of the two retailers. Both profits increase with the growing of demand disruption. The profit of retailer 1 participating in recovering the waste is always higher than that of retailer 2 in different cases of demand disruption. When the demand disruption is below zero, the profit difference between retailer 1 and retailer 2 is not significant. When the demand disruption is greater than zero and the positive disruption is larger, the increase of profit of retailer 2 tends to be stable, while the profit of retailer 1 is rising and the gap between the two retailers is widening. Therefore, retailers participating in recovering can expand market share and gain more profits under the disturbance, while retailers not participating in recovering can only maintain profits by increasing retail prices. Hence, retailers participating in collecting the waste can expand the market share faster and make more profits under disruption; by contrast, retailers not participating in recovering the scrapped products for remanufacturing can only maintain profits via increasing retail prices.

Figure 4 reflects the decision-making of retailer 1 . With the growth of demand, retailer 1 will increase investment in waste recovering. Therefore, the recovering rate will be on the rise. At the same time, retailer 1 regulates the demand of consumers for products by adjusting prices (as shown in Figure 2), to gain more profits or reduce the loss of benefits caused by demand disruption. In fact, when the recovering

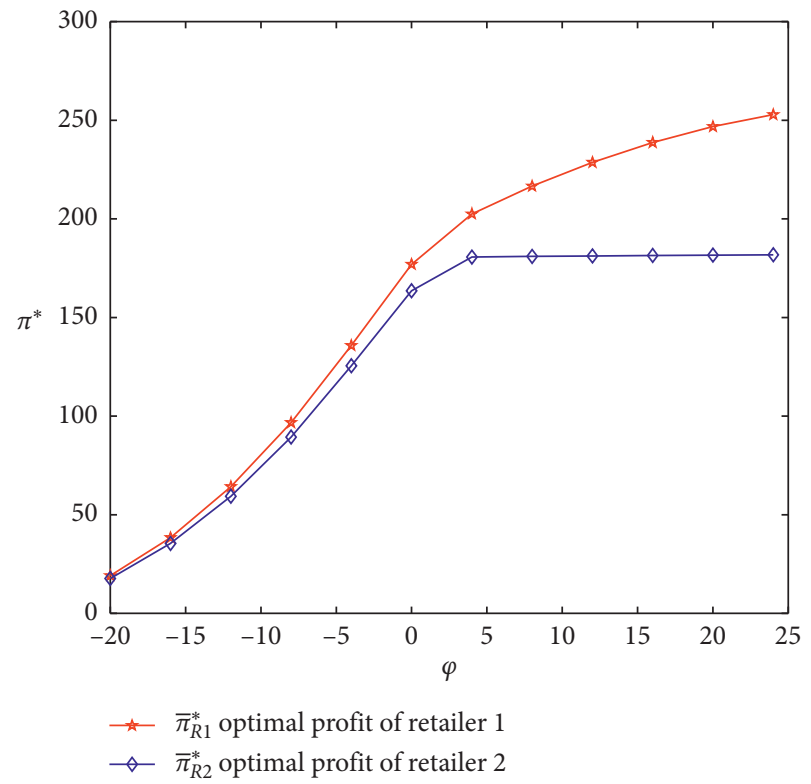

Figure 3: Comparison of optimal profits between two retailers.

rate reaches $100 \%$, a portion of retailer 1's rising profits comes from the recovering in waste products.

As is shown in Figure 5, when demand is disrupted positively, the manufacturer will pass on the additional costs, which arise from the production that exceed the expected output, by increasing the wholesale price. Therefore, the wholesale price of the manufacturer increases continuously under the influence of demand disruption, so does its profit. When demand is disrupted negatively, the products of manufacturer will be unsaleable. At this time, additional processing costs are necessary in order to deal with the redundancy of products. Therefore, the manufacturer must reduce the loss of unsalable products by lowering the 


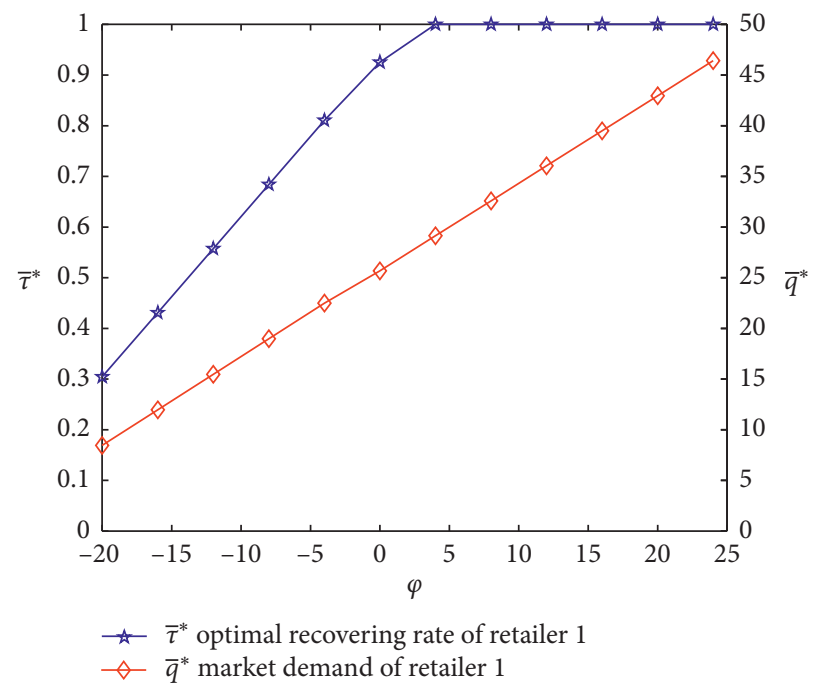

Figure 4: Optimal decision of retailer 1.

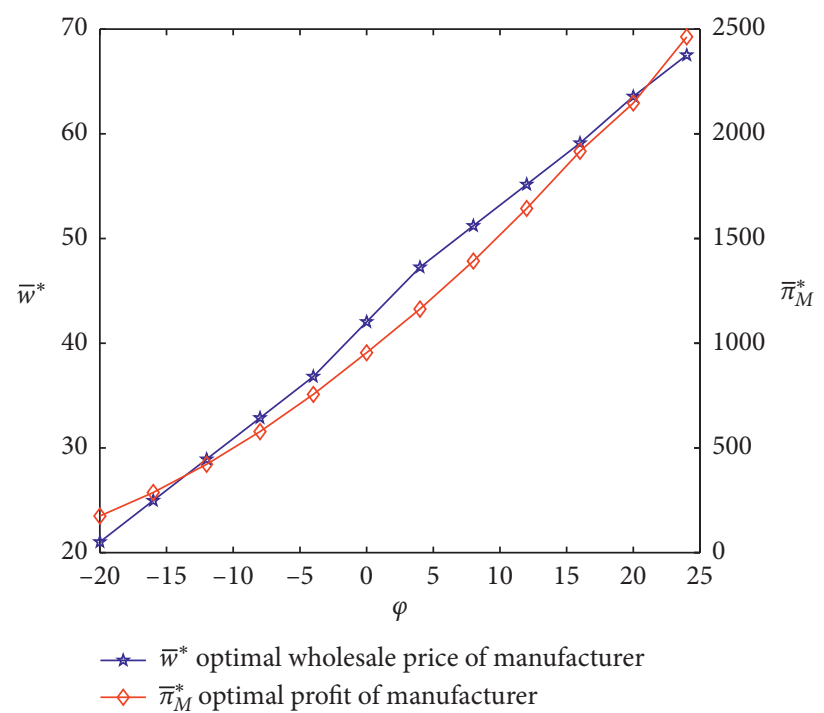

FIGURE 5: Optimal wholesale price and profit of the manufacturer.

TABLE 8: The range of demand disruption vs. the optimal profit of the manufacture.

\begin{tabular}{lc}
\hline Demand disruption $\varphi$ & Optimal profit of manufacture $\bar{\pi}_{M}^{*}$ \\
\hline $0<\varphi<(1-\beta) \lambda_{1}$ & $(h(1+\beta)(A+\phi)(A+\phi+2 \varphi)) /((\beta-1) B)$ \\
$(1-\beta) \lambda_{1} \leq \varphi \leq J$ & $\left(\left(h(1+\beta)\left[A+\lambda_{1}(\beta-1)+\phi+\varphi\right]\right) /((\beta-1) B)\right)+\lambda_{1} q^{*}$ \\
$J \leq \varphi \leq K$ & $\left.(2 h(1+\beta)) / \Delta[((2-\beta)(1+\beta) h) /((\beta-1) \Delta))+((\phi+\varphi) /(1-\beta))-c_{n}+(\Delta / 2)\right]$ \\
$\varphi \geq K$ & $\left(\left[\bar{b}^{*}\left(\beta^{2}-1\right)+2 V-2 \Delta(\beta-1)+2 \varphi\right] /(8(\beta-2)(\beta-1))\right)+\lambda_{1} q^{*}$ \\
\hline
\end{tabular}

wholesale price, and the profit of the manufacturer will also be reduced.

\section{Conclusions}

Notwithstanding the great progress achieved in the research of CLSC, scholars in various regions still have limited research on the operation of closed-loop supply chain under demand disturbance. The actual operation of supply chain is much more complex than that of the theoretic study. We construct a closed-loop supply chain consisting of a single manufacturer and two retailers; one of the retailers is participating in recovering waste products, while the other is not. Game theory and optimization theory are adopted to solve the optimal decisionmaking under both situations of demand stability and demand disruption. Main conclusions are summarized as follows. 
Firstly, by analyzing the changes of marketing price and other factors under the positive and negative demand disruptions, the optimal emergency decision-making of supply chain members under the different disturbances is investigated. The manufacturer will maintain a stable recovering compensation price under demand disruptions. The optimal wholesale price and the profit are positively correlated with the demand disruption. Two retailers develop their own optimal strategies based on the manufacturer's decisionmaking.

Secondly, by comparing the optimal decision-making of two retailers under demand disruption, the impact of retailers' participation in recovering the waste product on their market competitiveness is discussed. Compared with the retailers who do not take part in recovering, the retailers who decide to participate in recovering activities can expand market share faster and gain more profits under demand disruption. Thus, these retailers are more competitive in the market.

Thirdly, when the demand disruption occurs, the manufacturer adjusts the wholesale price of the product according to the direction of the demand disruption, and the recovery price remains unchanged. At the same time, the retailer adjusts the retail price. Because of the strong robustness of order quantity and recovering rate of waste products, they should be adjusted according to the disruption direction only when the disruptions of demand exceed a certain threshold. Moreover, the greater the positive demand disruption, the more conducive it is to carry out waste product recovering, which can not only enable consumers to get preferential treatment but also reuse waste products to reduce production costs, thereby continuously improving the overall welfare of society.

There is still room for future research. We only study the mode of single retailer participating in recovery of the waste and not involve the model of the manufacturer entrusting multiple retailers to recover or multiple retailers and even third parties participating in collection together. Moreover, the impact of government incentive policies on waste product recovering is worth studying.

\section{Appendix}

\section{A. Proof of Proposition 1}

The profit functions of manufacturer and two retailers under decentralized decision-making are shown as follows:

$$
\begin{aligned}
& \pi_{M}(w, b)=\left[w-c_{n}+\tau(\Delta-b)\right]\left[2 \phi+(\beta-1)\left(p_{1}+p_{2}\right)\right] \\
& \pi_{R_{1}}\left(p_{1}, \tau\right)=\left(p_{1}-w\right)\left(\phi-p_{1}+\beta p_{2}\right) \\
&+\left[2 \phi+(\beta-1)\left(p_{1}+p_{2}\right)\right] b \tau-h \tau^{2}, \\
& \pi_{R_{2}}\left(p_{2}\right)=\left(p_{2}-w\right)\left(\phi-p_{2}+\beta p_{1}\right) .
\end{aligned}
$$

Take partial derivative of equations (A.2) and (A.3) with respect to retail price of retailer $1 p_{1}$, recovering rate $\tau$, and retail price of retailer $2 p_{2}$ :

$$
\begin{gathered}
\frac{\partial \pi_{R_{1}}\left(p_{1}, \tau\right)}{\partial p_{1}}=\phi-2 p_{1}+\beta p_{2}+w+(\beta-1) b \tau=0, \\
\frac{\partial \pi_{R_{2}}\left(p_{1}, \tau\right)}{\partial \tau}=b\left[2 \phi+(\beta-1)\left(p_{1}+p_{2}\right)\right]-2 h \tau=0, \\
\frac{\partial \pi_{R_{2}}\left(p_{2}\right)}{\partial p_{2}}=\phi-2 p_{2}+\beta p_{1}+w=0 .
\end{gathered}
$$

From equations (A.4)-(A.6), we can have

$$
\begin{aligned}
p_{1}(w, b) & =-\frac{2 h(2+\beta)(w+\phi)+b^{2}(-1+\beta)[w(-1+\beta)+(3+\beta) \phi]}{\left[2 h(-2+\beta)+b^{2}(-1+\beta)^{2}\right](2+\beta)}, \\
\tau(w, b) & =-\frac{2 b[w(-1+\beta)+\phi]}{2 h(-2+\beta)+b^{2}(-1+\beta)^{2}} \\
p_{2}(w, b) & =\frac{-2 h(2+\beta)(w+\phi)+b^{2}\left[w(-1+\beta)^{2}+\left(1-\beta^{2}\right) \phi\right]}{\left[2 h(-2+\beta)+b^{2}(-1+\beta)^{2}\right](2+\beta)} .
\end{aligned}
$$

Substitute equations (A.7)-(A.9) into the profit function of the manufacturer:

$$
\pi_{M}(w, b)=-\frac{4 h[w(-1+\beta)+\phi]\left\{-2 h\left(c_{n}-w\right)(-2+\beta)-b^{2}\left[w-c_{n}(-1+\beta)^{2}-w \beta^{2}-2 \phi\right]-2 b \Delta[w(-1+\beta)+\phi]\right\}}{\left[2 h(-2+\beta)+b^{2}(-1+\beta)^{2}\right]^{2}} .
$$


When $\partial \pi_{M}(w, b) / \partial w=0$, we have $w$ By substituting the values of $w$ into equation (A.10), we have

$$
\pi_{M}(b)=\frac{h[w(-1+\beta)+\phi]^{2}}{(-1+\beta)\left[2 h(-2+\beta)+b^{2}\left(-1+\beta^{2}\right)-2 b(-1+\beta) \Delta\right]} .
$$

The denominator of equation (A.11), which has a minimum value, is a quadratic function of $b$. When the denominator takes the minimum value $\left(b^{*}=\Delta /(\beta+1)\right)$, the formula reaches the maximum. Then, we have $b^{*}$ and $w^{*}$. By substituting them into $p_{1}$ and $p_{2}$, other parameters can be obtained as shown in Proposition 1.

\section{B. Proof of Lemma 1}

When $\varphi>0$, there are four possible relationships between $\bar{q}^{*}$ and $q^{*}$ and $\bar{\tau}^{*}$ and $\tau^{*}$ :
(i) $\bar{q}^{*} \geq q^{*}, \bar{\tau}^{*}<\tau^{*}$
(ii) $\bar{q}^{*} \geq q^{*}, 1 \geq \bar{\tau}^{*} \geq \tau^{*}$
(iii) $\bar{q}^{*}<q^{*}, 1 \geq \bar{\tau}^{*} \geq \tau^{*}$

(iv) $\bar{q}^{*}<q^{*}, \bar{\tau}^{*}<\tau^{*}$

In order to demonstrate the relationship among the optimal order quantity, optimal waste recovering rate, and demand disruption, Scenario I is discussed firstly.

The profit function of the manufacturer is

$$
\begin{aligned}
\bar{\pi}_{M}= & {\left[2(\phi+\varphi)+(\beta-1)\left(\bar{p}_{1}+\bar{p}_{2}\right)\right]\left[\bar{w}-c_{n}+\bar{\tau}(\Delta-\bar{b})\right] } \\
& -\lambda_{1}\left[2(\phi+\varphi)+(\beta-1)\left(\bar{p}_{1}+\bar{p}_{2}\right)-q^{*}\right] .
\end{aligned}
$$

Simplifying equation (B.1), we can get

$$
\begin{aligned}
\bar{\pi}_{M}= & {\left[2(\phi+\varphi)+(\beta-1)\left(\bar{p}_{1}+\bar{p}_{2}\right)\right] } \\
& \cdot\left[\bar{w}-c_{n}-\lambda_{1}+\bar{\tau}(\Delta-\bar{b})\right]+\lambda_{1} q^{*} .
\end{aligned}
$$

Taking partial derivative of equations (B.1) and (B.2) with respect to $\bar{p}_{1}, \bar{p}_{2}$, and $\bar{\tau}$, we have the optimal retail price of two retailers and the optimal recycling rate of retailer 1 on $\bar{w}$ and $\bar{b}$ :

$$
\begin{aligned}
& \bar{p}_{1}^{*}(\bar{w}, \bar{b})=\frac{\bar{b}^{2}(\beta-1)[\bar{w}(\beta-1)+(3+\beta)(\phi+\varphi)]-2 h(2+\beta)(\bar{w}+\phi+\varphi)}{\left[2 h(-2+\beta)+\bar{b}^{2}(-1+\beta)^{2}\right](2+\beta)}, \\
& \bar{p}_{2}^{*}(\bar{w}, \bar{b})=\frac{\bar{b}^{2}\left[\bar{w}(\beta-1)^{2}-\left(\beta^{2}-1\right)(\phi+\varphi)\right]-2 h(2+\beta)(\bar{w}+\phi+\varphi)}{\left[2 h(\beta-2)+\bar{b}^{2}(\beta-1)^{2}\right](2+\beta)}, \\
& \bar{\tau}^{*}(\bar{w}, \bar{b})=\frac{-2 \bar{b}[\bar{w}(\beta-1)+\phi+\varphi]}{2 h(\beta-2)+\bar{b}^{2}(\beta-1)^{2}} .
\end{aligned}
$$

By substituting equations (B.3)-(B.5) into equation (B.2), the problem can be simplified as follows.

$$
\begin{aligned}
& \max \bar{\pi}_{M}=\frac{-4 h[\bar{w}(\beta-1)+\phi+\varphi]}{2 h(\beta-2)+\bar{b}^{2}(\beta-1)^{2}} \times\left\{\bar{w}-c_{n}-\lambda_{1}+(\Delta-\bar{b}) \cdot \frac{-2 \bar{b}[\bar{w}(\beta-1)+\phi+\varphi]}{2 h(\beta-2)+\bar{b}^{2}(\beta-1)^{2}}\right\}+\lambda_{1} q^{*} \\
& \text { s.t. } q^{*}-\bar{q}^{*} \leq 0 \\
& \qquad \bar{\tau}^{*}<\tau^{*}
\end{aligned}
$$

By introducing Lagrange multiplier $\gamma=0$ and relaxing the constraint equations (B.7) and (B.8), the following KKT conditions can be obtained. 


$$
\left\{\begin{array}{l}
\frac{-4 h[\bar{w}(-1+\beta)+\phi+\varphi]}{2 h(-2+\beta)+\bar{b}^{2}(-1+\beta)^{2}} \times\left[1-\frac{(\Delta-\bar{b}) 2 \bar{b}(-1+\beta)}{2 h(-2+\beta)+\bar{b}^{2}(-1+\beta)^{2}}\right]-\frac{4 h(-1+\beta) \gamma}{2 h(-2+\beta)+\bar{b}^{2}(-1+\beta)^{2}} \\
+\left\{\bar{w}-c_{n}-\lambda_{1}+(\Delta-\bar{b}) \frac{-2 \bar{b}[\bar{w}(-1+\beta)+\phi+\varphi]}{2 h(-2+\beta)+\bar{b}^{2}(-1+\beta)^{2}}\right\} \frac{-4 h(-1+\beta)}{2 h(-2+\beta)+\bar{b}^{2}(-1+\beta)^{2}}=0, \\
\gamma\left\{-\frac{-4 h[\bar{w}(-1+\beta)+\phi+\varphi]}{2 h(-2+\beta)+\bar{b}^{2}(-1+\beta)^{2}}+\frac{-2 h(1+\beta)\left[c_{n}(-1+\beta)+\phi\right]}{2 h\left(-2+\beta+\beta^{2}\right)-(-1+\beta) \Delta^{2}}\right\}=0, \\
\gamma \geq 0,
\end{array}\right.
$$

(1) When $\gamma=0$, the optimal wholesale price and profit of the manufacturer are

$$
\begin{aligned}
& \bar{w}^{*}=\frac{2 h(\beta-2)(V-2 \phi-\varphi)+4 \bar{b}(\beta-1) \Delta(\phi+\varphi)+\bar{b}^{2}(\beta-1)\left[\left(c_{n}+\lambda_{1}\right)(\beta-1)^{2}-(3+\beta)(\phi+\varphi)\right]}{2(\beta-1)\left[2 h(\beta-2)+\bar{b}^{2}\left(\beta^{2}-1\right)-2 \bar{b}(\beta-1) \Delta\right]}, \\
& \bar{\pi}_{M}^{*}=\frac{h(V+\varphi)^{2}}{(\beta-1)\left[2 h(\beta-2)+\bar{b}^{2}\left(\beta^{2}-1\right)-2 \bar{b}(\beta-1) \Delta\right]}+\lambda_{1} q^{*} .
\end{aligned}
$$

The denominator of equation (B.11), which has a minimum value, is a quadratic function of $\bar{b}$. When the denominator takes the minimum value $\left(\bar{b}^{*}=\Delta / \beta+1\right)$, the formula reaches to the maximum. Then, we have the optimal profit $\pi_{M}^{*}$, orders $\bar{q}^{*}$, and recycling rate $\bar{\tau}^{*}$ of the manufacture, which are as presented follows: $\bar{\pi}_{M}^{*}=((h(1+$ $\left.\left.\beta)(V+\varphi)^{2}\right) /((1-\beta) B)\right)+\lambda_{1} q^{*}, \bar{q}^{*}=(2 h(1+\beta)[V+\varphi]) /-$ $B, \bar{\tau}^{*}=\Delta[V+\varphi] / B$.

According to the scenario I, when $\varphi>0$, we have $\bar{q}^{*}>q^{*}$ and $\bar{\tau}^{*}<\tau^{*}$. By substituting the upper values into $\bar{q}^{*}>q^{*}$ and $\bar{\tau}^{*}<\tau^{*}$, it can be obtained that $\varphi \leq(1-\beta) \lambda_{1}$ and $\varphi>(1-\beta) \lambda_{1}$. As we cannot get a feasible solution, the conclusion is drawn that the scenario (1) has no solution when $\gamma=0$.

(2) When $\gamma \neq 0, q^{*}$ meets the conditions as follows:

$$
q^{*}-\frac{-4 h\left[\bar{w}^{*}(\beta-1)+\phi+\varphi\right]}{2 h(\beta-2)+\bar{b}^{2}(\beta-1)^{2}}=0
$$

Then, we have the optimal profit of the manufacture from equation (B.12):

$$
\bar{\pi}_{M}^{*}=\frac{h(1+\beta)(A+\phi)\left[\bar{b}^{* 2}(1-\beta)\left(1+\beta^{2}\right)(A+\phi)+2 \bar{b}^{*} \Delta\left(\beta^{2}-1\right)(A+\phi)-2 D(A+\phi+\varphi)+h\left(\beta^{2}-\beta-2\right)(A+\phi+2 \varphi)\right]}{(\beta-1) B^{2}} .
$$

Equation (B.13) is a quadratic equation of one variable $B$. When $\bar{b}^{*}=\Delta / \beta+1$, the equation takes the maximum value as follows:

$$
\bar{\pi}_{M}^{*}=\frac{h(1+\beta)\left[c_{n}(\beta-1)+\phi\right](A+\phi+2 \varphi)}{(1-\beta) B} .
$$

To sum up, we can get the range of demand disruption in Scenario I as $0<\varphi<(1-\beta) \lambda_{1}$.

Similarly, the range of demand disruption and the optimal profit of the manufacture in Scenario $\Pi$ are shown in Table 8:

Similarly, there is no feasible solution for Scenarios Ш and IV. Thus, when an emergency causes demand 
disruption, if $\varphi>0$, then $\bar{q}^{*} \geq q^{*}, 1 \geq \bar{\tau}^{*} \geq \tau^{*}$; if $\varphi<0$, then $\bar{q}^{*} \leq q^{*}, \bar{\tau}^{*} \leq \tau^{*}$.

\section{Data Availability}

The data used to support the findings of this study are included within the article.

\section{Conflicts of Interest}

The authors declare that there are no conflicts of interest regarding the publication of this paper.

\section{Acknowledgments}

This research work was supported by the National Natural Science Foundation of China (71874159 and 71371169) and the Natural Science Foundation of Zhejiang, China (LY18G020020).

\section{References}

[1] J.-Z. Huo, Y.-T. Hou, F. Chu, and J.-K. He, "A combined average-case and worst-case analysis for an integrated hub location and revenue management problem," Discrete Dynamics in Nature and Society, vol. 2019, pp. 1-13, 2019.

[2] S. D. Miao, D. Y. Chen, and T. F. Wang, "System dynamics modelling of remanufacturing and recycling mode based on closed-loop across-chain competition," Discrete Dynamics in Nature and Society, no. 12, Article ID 8152365, 2017.

[3] S. Pulvirenti, P. Pavone, R. A. Carbonaro, and R. M. S. Costa, "Taxonomic study of the plants to be found in the onlyherbariumof Paolo Boccone (1633-1704) at present existing in Italy," Plant Biosystems-An International Journal Dealing with All Aspects of Plant Biology, vol. 151, no. 4, pp. 745-759, 2017.

[4] S. Pulvirenti, P. Pavone, R. A. Carbonaro, and R. M. S. Costa, "The controversial biography of paolo boccone (1633-1704) and his "grand tour" from the mediterranean to northern europe," Plant Biosystems-An International Journal Dealing with All Aspects of Plant Biology, vol. 151, no. 3, pp. 377-380, 2017.

[5] R. C. Savaskan, S. Bhattacharya, and L. N. Van Wassenhove, "Closed-loop supply chain models with product remanufacturing," Management Science, vol. 50, no. 2, pp. 239-252, 2004.

[6] J. Cao, X. Chen, X. Zhang, Y. Gao, X. Zhang, and S. Kumar, "Overview of remanufacturing industry in China: government policies, enterprise, and public awareness," Journal of Cleaner Production, vol. 242, p. 118450, 2020.

[7] E. Revilla and M. J. Sáenz, "Supply chain disruption management: global convergence vs national specificity," Journal of Business Research, vol. 67, no. 6, pp. 1123-1135, 2014.

[8] M. A. Cohen and H. Kunreuther, "Operations risk management: overview of Paul Kleindorfer's contributions," Production and Operations Management, vol. 16, no. 5, pp. 525-541, 2007.

[9] A. M. Knemeyer, W. Zinn, and C. Eroglu, "Proactive planning for catastrophic events in supply chains," Journal of Operations Management, vol. 27, no. 2, pp. 141-153, 2009.

[10] G.-f. Yang, Z.-p. Wang, and X.-q. Li, "The optimization of the closed-loop supply chain network," Transportation Research Part E: Logistics and Transportation Review, vol. 45, no. 1, pp. 16-28, 2009.
[11] J. Shi, G. Zhang, and J. Sha, "Optimal production planning for a multi-product closed loop system with uncertain demand and return," Computers \& Operations Research, vol. 38, no. 3, pp. 641-650, 2011.

[12] T.-M. Choi, Y. Li, and L. Xu, "Channel leadership, performance and coordination in closed loop supply chains," International Journal of Production Economics, vol. 146, no. 1, pp. 371-380, 2013.

[13] M. Huang, M. Song, L. H. Lee, and W. K. Ching, "Analysis for strategy of closed-loop supply chain with dual recycling channel," International Journal of Production Economics, vol. 144, no. 2, pp. 510-520, 2013.

[14] J. D. Abbey, M. G. Meloy, V. D. R. Guide, and S. Atalay, "Remanufactured products in closed-loop supply chains for consumer goods," Production and Operations Management, vol. 24, no. 3, pp. 488-503, 2015.

[15] P. De Giovanni, P. V. Reddy, and G. Zaccour, "Incentive strategies for an optimal recovery program in a closed-loop supply chain," European Journal of Operational Research, vol. 249 , no. 2 , pp. 605-617, 2016.

[16] J. Gaur, M. Amini, and A. K. Rao, "Closed-loop supply chain configuration for new and reconditioned products: an integrated optimization model," Omega, vol. 66, pp. 212-223, 2017.

[17] M. Reimann, Y. Xiong, and Y. Zhou, "Managing a closed-loop supply chain with process innovation for remanufacturing," European Journal of Operational Research, vol. 276, no. 2, pp. 510-518, 2019.

[18] J. Clausen, J. Hansen, J. Larsen, and A. Larsen, "Disruption management," OR/MS Today, vol. 38, no. 5, pp. 40-43, 2001.

[19] X. Qi, J. F. Bard, and G. Yu, "Supply chain coordination with demand disruptions," Omega, vol. 32, no. 4, pp. 301-312, 2004.

[20] P. R. Kleindorfer and G. H. Saad, "Managing disruption risks in supply chains," Production and Operations Management, vol. 14, no. 1, pp. 53-68, 2005.

[21] T. J. Xiao, X. T. Qi, and G. Yu, "Coordination of supply chain after demand disruptions when retailers compete," International Journal of Production Economics, vol. 109, no. 1-2, pp. 162-179, 2007.

[22] J. Hou, A. Z. Zeng, and L. D. Zhao, "Coordination with a backup supplier through buy-back contract under supply disruption," Transportation Research Part E-Logistics and Transportation Review, vol. 46, no. 6, pp. 881-895, 2010.

[23] S. Huang, C. Yang, and X. Zhang, "Pricing and production decisions in dual-channel supply chains with demand disruptions," Computers \& Industrial Engineering, vol. 62, no. 1, pp. 70-83, 2012.

[24] S. Huang, C. Yang, and H. Liu, "Pricing and production decisions in a dual-channel supply chain when production costs are disrupted," Economic Modelling, vol. 30, pp. 521-538, 2013.

[25] B. C. Giri and S. Sharma, "Optimal production policy for a closed-loop hybrid system with uncertain demand and return under supply disruption," Journal of Cleaner Production, vol. 112, pp. 2015-2028, 2016.

[26] Y. Huang and Z. Wang, "Dual-recycling channel decision in a closed-loop supply chain with cost disruptions," Sustainability, vol. 9, no. 11, 2017.

[27] Y. Huang and Z. Wang, "Demand disruptions, pricing and production decisions in a closed-loop supply chain with technology licensing," Journal of Cleaner Production, vol. 191, pp. 248-260, 2018. 